\title{
Three Dimensional Visualization of Gas Evolution and Channel Formation inside a Lithium-ion Battery ${ }^{\#}$
}

\author{
Fu Sun*, ${ }^{, \dagger \neq}$, Henning Markötter ${ }^{\dagger, \neq}$, Ingo Manke ${ }^{\ddagger}$, Andre Hilger ${ }^{\ddagger}$, Nikolay Kardjilov ${ }^{\ddagger}$ and \\ John Banhart ${ }^{\dagger, \ddagger}$ \\ ${ }^{\dagger}$ Institute of Materials Science and Technology \\ Technische Universität Berlin \\ 10623 Berlin, Germany \\ ${ }^{\ddagger}$ Helmholtz Centre Berlin for Materials and Energy \\ Hahn-Meitner-Platz 1 \\ 14109 Berlin, Germany \\ *Corresponding Author: fu.sun@helmholtz-berlin.de \\ \#ACS Applied Materials \& Interfaces 8(11), 7156-7164 (2016) \\ American Chemical Society, ISSN: 1944-8244 — doi: 10.1021/acsami.6b00708
}

\begin{abstract}
Gas generation within lithium ion batteries (LIBs) gives rise to safety concerns that question their applicability. By employing synchrotron X-ray imaging, the gas and channel evolution occurring in an operating LIB have been directly visualized in their inherent 3D state as a function of discharge and charge. Using the spatial 3D distribution of gas bubbles and channels, the active particles that dictate the performance of a functional LIB were identified and visualized in 3D. Delithiation and lithiation are interpreted as the process of activating particles continuously in a step-bystep way. The present work not only demonstrates the generation and evolution of gas within LIB in 3D, but also reveals the distribution of active particles for the first time. These fundamentally findings presented here shed light on a range of processes that could not previously be characterized in 3D and can provide practical guidance for the
\end{abstract}

\section{INTRODUCTION}

Rechargeable lithium-ion batteries (LIBs) have penetrated profoundly into products such as portable electronic devices, electric vehicles or other large-sized power sources. ${ }^{1-5}$ However, safety concerns still limit the full practical utilization of these batteries. ${ }^{6}$ Especially gas evolution is a formidable technological and fundamental challenge ${ }^{7}$ since gas generated in hermetically sealed batteries can lead to detrimental effects: on the one hand, gas generation during storage results in a diminished shelf life and eventually a markedly reduced cycle lifetime. ${ }^{8}$ On the other hand, gas evolution during cycling leads to electrolyte displacement, ${ }^{9}$ which causes a decrease of Li-ion diffusion and/or Li-ion conduction in the electrolyte and ultimately contributes to a tremendous increase of battery resistance. ${ }^{10}$ In both cases, the internal pressure build-up may also induce battery bulging, mechanical stress inside the electrodes and even severe gas leakage, ${ }^{11}$ all of which are detrimental to longevity and reliability of energy storage battery systems. Therefore, understanding the gas evolution mechanisms are of great importance from a practical point of view. 
To date, many experimental investigations have been carried out in extensive detail and, as a result, our knowledge of the gas evolution mechanism has substantially deepened. For instance, it is already known that the gas evolution, which is intimately related with solid electrolyte interphase (SEI) formation in the electrochemical battery, ${ }^{12}$ originates from reductive and oxidative electrolyte decomposition reactions due to the fact that the electrochemical potential of both electrode materials is far beyond the thermodynamic stability window of the commonly used organic electrolytes. ${ }^{13}$ Actually, significant evolution of gaseous products such as $\mathrm{CO}_{2}, \mathrm{CO}, \mathrm{O}_{2}, \mathrm{H}_{2}, \mathrm{CH}_{4}, \mathrm{C}_{2} \mathrm{H}_{4}, \mathrm{C}_{2} \mathrm{H}_{6}, \mathrm{C}_{3} \mathrm{H}_{6}$ and $\mathrm{C}_{3} \mathrm{H}_{8}$ from the decomposition of carbonate solvents and lithium during battery operation has been detected by various characterization techniques. ${ }^{14-21}$ In addition, utilizing isotope analysis, Onuk et al. have unambiguously identified the origin of gases evolving in LIBs. ${ }^{22}$ Furthermore, by adopting in situ transmission electron microscopy (TEM) ${ }^{23}$ and neutron radiography (NR), ${ }^{9,11}$ the generation of gaseous bubbles channels formed by the gas have recently been visualized. These studies have enhanced our understanding of gas evolution but, unfortunately, the applied radiographic imaging techniques yield two-dimensional (2D) information and do not help us in further comprehending gas evolution kinetics in its inherently three-dimensional (3D) state and in quantitatively analyzing its complex evolution. Moreover, no study of the spatial gas distribution in relation to the electrochemically active particle population ${ }^{24}$ in electrodes has been conducted so far, even though it is the active particles that directly determine battery performance and cycle life. ${ }^{25}$

Due to the high fluxes generated by synchrotron sources, synchrotron X-ray imaging has evolved into a powerful characterization tool in the field of materials science. ${ }^{26-36}$ Especially synchrotron X-ray tomography has enabled researchers to obtain unprecedented insights into LIBs from the level of individual particles to the scale of entire electrodes. ${ }^{37-40}$ Most recently, Ebner et al., have directly observed and quantified electrochemical and mechanical degradation in a $\mathrm{SnO}$ anode. ${ }^{41}$

Using a non-destructive 3D X-ray imagining technique, herein we investigate in 3Ds the gas evolution scenario in a LIB based on a silicon ( $\mathrm{Si}$ ) electrode since $\mathrm{Si}$ is considered one of the most promising anode materials for next-generation power sources. ${ }^{42-43}$ This is the first study that also visualizes the distribution of electrochemically active electrode particles with respect to the spatial gas development and interprets delithiation and lithiation as a process of activating particles in a step-by-step way. This work sets a refined example for the 3D gas evolution investigation and the spatially evolved active particle distribution, providing indepth knowledge for the electrode engineers and numerical simulation experts.

\section{EXPERIMENTAL SECTION}

Materials. Active silicon particles were received from Elkem AS, Norway. Conductive carbon black, Polyvinylidene fluoride (PVDF) binder, Celgard separator and lithium were purchased from MTI Cor. USA. N-methyl pyrrolidone solvent (NMP) and $1 \mathrm{M} \mathrm{LiPF}_{6}$ in a volume-ratio (1:1) mixture of ethylene carbonate (EC) and dimethyl carbonate (DMC) were purchased from Sigma Aldrich. The housing of the proof-of-concept beamline battery is made of polyamide-imide (Torlon), from McMaster-Carr.

Materials characterization. Scanning electron microscopy (SEM) images were taken using a Zeiss ultraplus microscope. The particle size distribution of Si particles was measured by laser diffraction using a Malvern Mastersizer 2000 analyzer.

Battery preparation. The electrode is made of electrode slurries with weight ratios of Si:carbon black:binder of 80:10:10 in NMP. The slurry was cast directly onto the head of the bottom screw shown in Figure 1. To remove the NMP, the cast slurry was dried in an oven at $60{ }^{\circ} \mathrm{C}$ overnight. Before and after casting, the screw was weighed to determine the mass of the 
electrode materials. The proof-of-concept battery (shown in Figure 1a) designed for beamline use was assembled in an argon filled glovebox with humidity and oxygen levels below 0.1 ppm. Metallic lithium was placed onto the head of the top screw in Figure 1a acting as both a counter and reference electrode. A polymer separator soaked with the electrolyte was placed between the lithium electrode and the $\mathrm{Si}$ electrode. Current leads are connected to a potentiostat for electrochemical tests.

Electrochemical measurements. Cyclic voltametry (CV) and galvanostatic charge/discharge of the battery were carried out using an IviumStat voltameter. A fresh battery after assembly was measured for $\mathrm{CV}$ curves from $0 \mathrm{~V}$ to $2.5 \mathrm{~V}$ at a scan rate of 1 $\mathrm{mV} / \mathrm{s}$. During galvanostatic cycling at the beamline, the assembled battery was measured at $1.75 \mathrm{Ag}^{-1}$ based on the mass of active silicon. Different discharge and charge current rates will be used to further investigate the correlation between the current rate and the gas releasing and channel formation behavior.

Settings of tomography and radiography. Synchrotron X-ray tomography and radiography were recorded at the BAMline at the storage ring BESSY II of the HelmholtzZentrum Berlin, Germany. The synchrotron beam was monochromatized to $20 \mathrm{KeV}$ using a double multilayer monochromator with an energy resolution of about $1.5 \%$. The detector system comprised a $60-\mu \mathrm{m}$ thick $\mathrm{CdWO}_{4}$ scintillator, a microscopic optic and a pco4000 camera with a $4008 \times 2672$ pixel $^{2}$ CCD chip that is kept out of the direct beam by using a mirror. For tomography, 2200 projections during a $180^{\circ}$ battery rotation each with $4 \mathrm{~s}$ exposure time were recorded before/after discharge/charge. The battery was radiographically imaged during discharge and charge with $4 \mathrm{~s}$ exposure time. $4 \mathrm{~s}$ exposure time was required because of the relative low photon flux provided by the BAMline compared to other imaging stations. It has to be noted that in order to characterize the morphological change of the whole Si based electrode (3.2 $\mathrm{mm} \times 0.4 \mathrm{~mm}$, length $\times$ width), we have used an optic and camera combination with a corresponding field of view of $3.3 \mathrm{~mm} \times 2.2 \mathrm{~mm}$. And the resultant voxel resolution is $\sim 0.876 \mu \mathrm{m}$. However, -10 er camera system with higher resolution of $\sim 0.438 \mu \mathrm{m}$ will be used in future investigations.

Data processing. Filtering, binarization and segmentation was performed in ImageJ and Avizo Fire. Data visualization was done using VGStudio MAX and Avizo Fire. Analysis of the particles is conducted by ImageJ. The procedure is presented in Supporting Information.

\section{RESULTS AND DISCUSSION}

An electrochemical cell that can fully represent a working battery and at the same time is compatible with synchrotron X-ray imaging is crucial for the characterization of gas evolving during operation. For this purpose, we developed an electrochemical cell that is X-ray transparent so that imaging can be conducted in operando to directly study gas evolution during dis/charge processes, allowing us to correlate the gas evolution with electrochemical activity. Figure 1 displays a photograph and the corresponding schematic illustration of the battery as well as a schematic representation of the synchrotron X-ray tomography setup. ${ }^{44}$ 


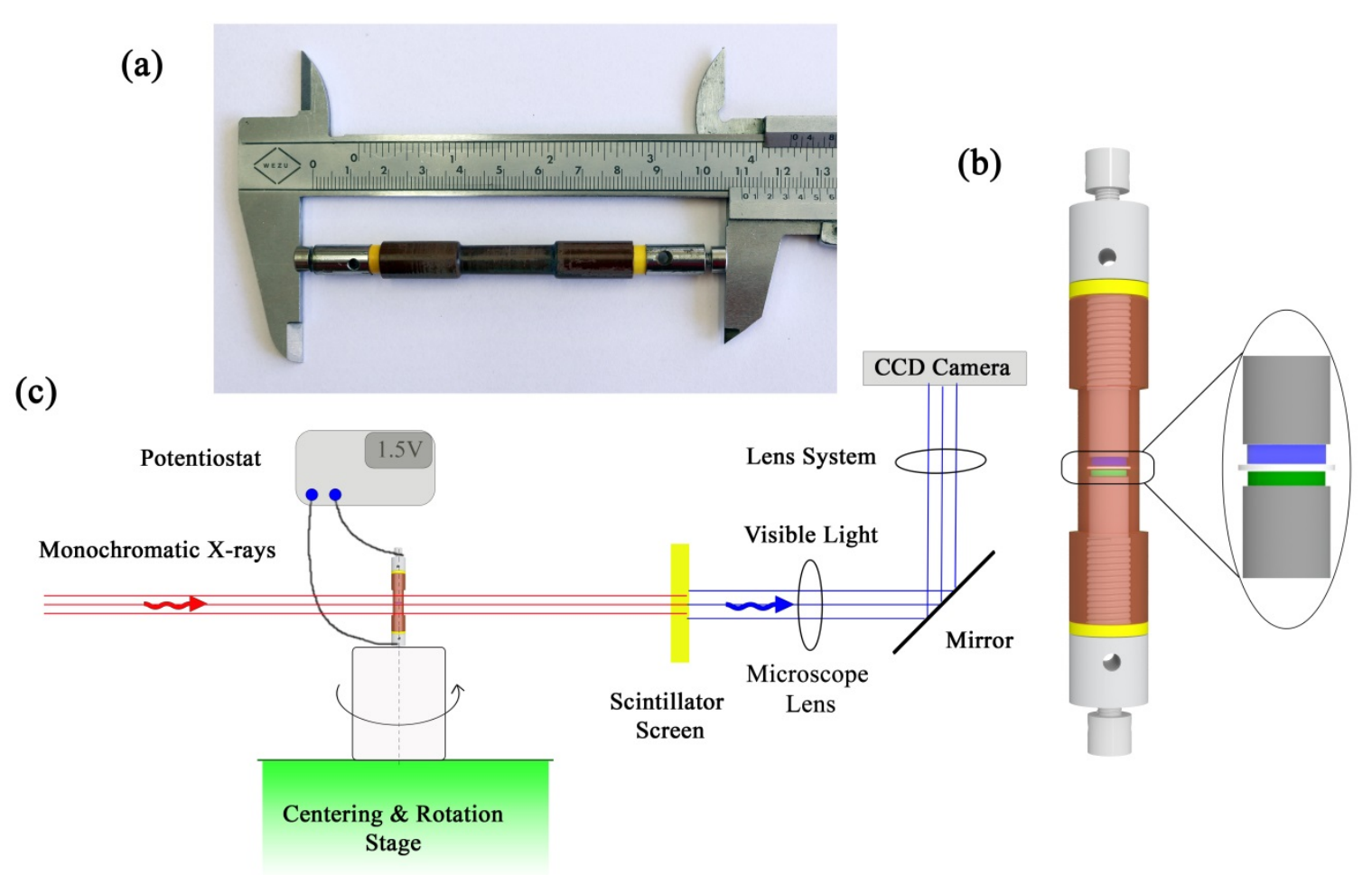

Figure 1. The customized electrochemical battery and the employed X-ray tomography setup: a) Photograph of the battery investigated. b) Corresponding schematic representation of the battery cell consisting of a polyamide-imide housing (brown), two screw electrodes and a retaining screws each on top (light grey), two sealing rings (yellow), a lithium metal electrode (blue), a porous separator (white) and the Si-based anode (green). c) Schematic representation of the experimental setup of the tomography station at the BAMline at BESSY II, HelmholtzZentrum Berlin, Germany

After assembly of the battery, cyclic voltametry (CV) was performed to verify the reduction and oxidation characteristics of silicon. Figure 2a shows CV curves of the battery scanned at a rate of $1 \mathrm{mVs}^{-1}$ in the potential window of $0-2.5 \mathrm{~V}$. The cathodic current increase from $1.4 \mathrm{~V}$ to $0.7 \mathrm{~V}$ in the $\mathrm{CV}$ curves is related to small-scale reduction of electrolyte and/or surface contaminations such as trace water. ${ }^{45}$ The small hump around $0.5 \mathrm{~V}$ is attributed to a strong reductive decomposition of the electrolyte associated to the release of large amounts of gases, ${ }^{46}$ while the peak above $0 \mathrm{~V}$ is related to $\mathrm{Li}$ alloying with $\mathrm{Si}$. During delithiation, the anodic peak observed slightly above $0.5 \mathrm{~V}$ is for de-alloying of Li-Si phase and the broad anodic peak at $1.0 \mathrm{~V}$ is related to the oxidation of electrolyte and/or compounds previously reduced during the cathodic process. The results are in good agreement with previously reported $\mathrm{Si} /$ carbon composites, ${ }^{43,47}$ thus confirming the electrochemical reactions inside the battery. 
(a)

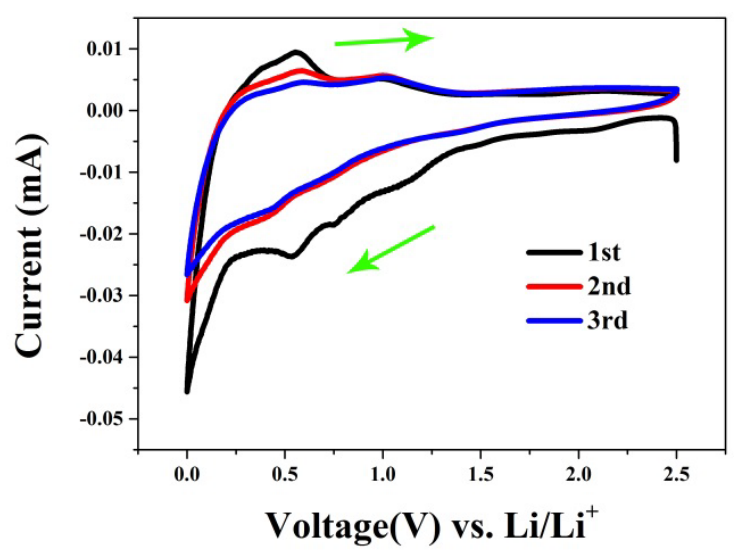

(b)

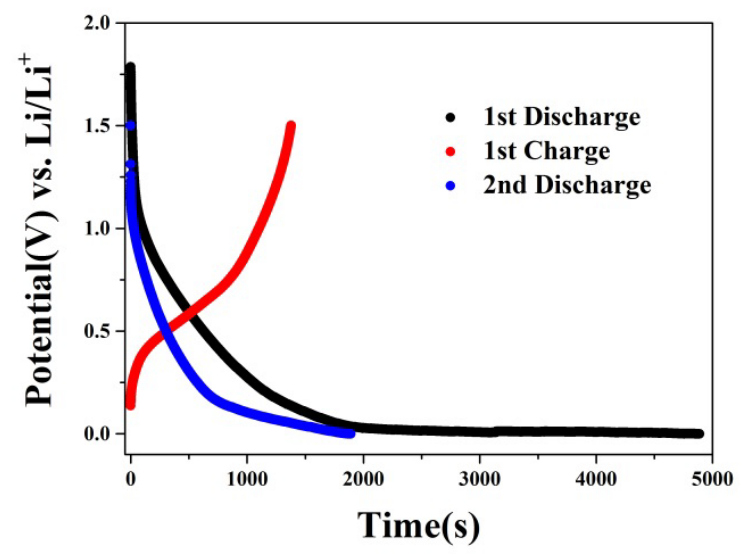

Figure 2. Electrochemical characterization of the investigated battery: a) CV curves of the first three cycles of the battery at a scan rate of $1 \mathrm{mVs}^{-1}$. b) The first discharge, the first charge and the second discharge voltage-time profiles of the battery at $1.75 \mathrm{Ag}^{-1}$ rate.

After this, we conducted X-ray radiography while simultaneously galvanostatically discharging or charging the cell at a rate of $1.75 \mathrm{Ag}^{-1}$ as shown in Figure 2b. The discharge and charge curves are also in good agreement with previous results for Si electrodes. ${ }^{43}$ In addition, another battery was galvanostatically discharged/charged at a small current of $0.01 \mathrm{Ag}^{-1}$ for more than $20 \mathrm{~h}$ as shown in Supporting Information (SI) Figure S1, showing typical characteristic features of Si. Thus, the battery design used for tomographic measurements exhibits an authentic electrochemical behavior.

High-resolution synchrotron X-ray tomographic imaging grants us the ability to probe the internal structure and the distribution of elements in the electrode nondestructively. ${ }^{40}$ First, the pristine battery without being exposed to any discharge or charge is characterized. The typical procedure of tomographic data acquisition and processing is demonstrated in Figure 3. Figure 3a shows a projection image of the Li electrode/separator/Si electrode assembly. While the Li electrode and the porous plastic separator appear nearly invisible due to their low X-ray absorption coefficients, the Si electrode is clearly visible. As the battery rotates during tomography, a series of projections are recorded, which are later reconstructed into a three dimensional volume. Figure $3 \mathrm{~b}$ shows a horizontal slice image through the reconstructed volume, which displays that the Si particles are well mixed with the carbon black. In order to further analyze the Si particles, the grayscale slices were segmented into binary images, yielding Figure 3c, in which the bright regions correspond to higher-absorbing material, the $\mathrm{Si}$ particles, whereas the black regions are assigned to less-absorbing material such as carbon black and polymer binder. Segmentation is performed via a combined approach of filtering and thresholding ${ }^{48}$ and produces Figure $3 \mathrm{~d}$ after labeling. In order to validate the X-ray tomography results qualitatively and quantitatively we compare scanning electron microscopy images (SEM) (Figure 3e) with grayscale slice images as well as the particle size distribution (PSD) measured using laser diffraction with the PSD obtained from the tomographic data (Figure 3f). The good agreement between the experimentally and numerically obtained results indicates that the segmentation applied accurately captures the electrode material properties. Finally, a full-view 3D representation of the segmented particles is shown in Figure $3 \mathrm{~g}$ and in the Supporting Movie 1 (SM 1) (More details can be found in the SI.) 


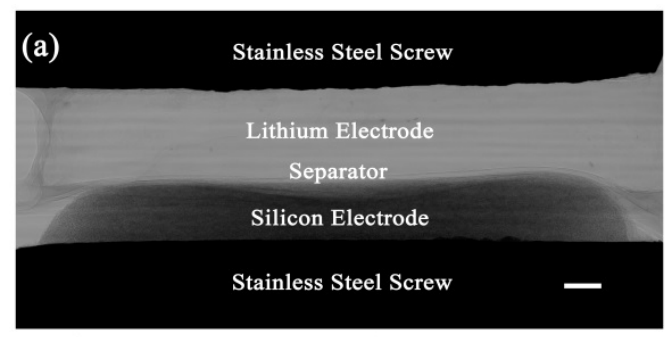

(d)

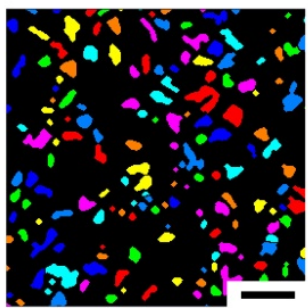

(b)

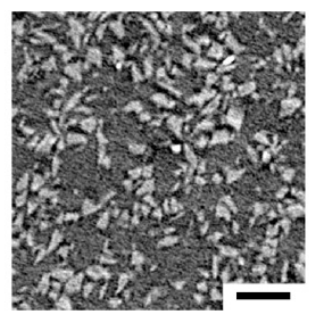

(c)

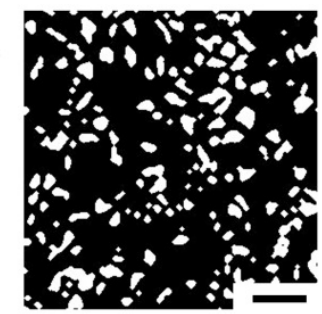

(e)

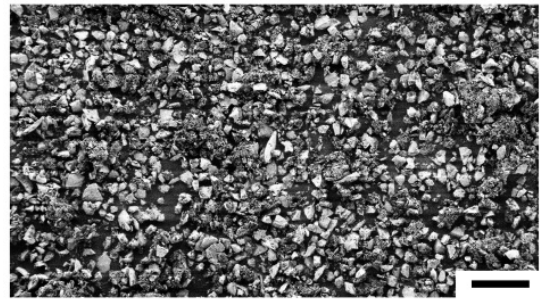

(f)

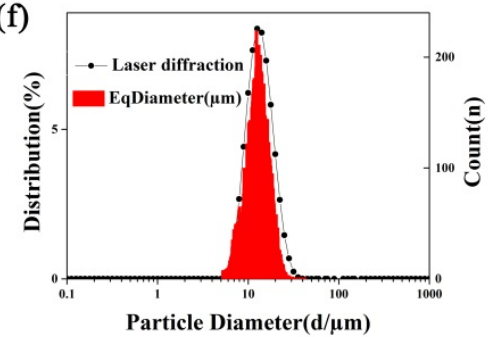

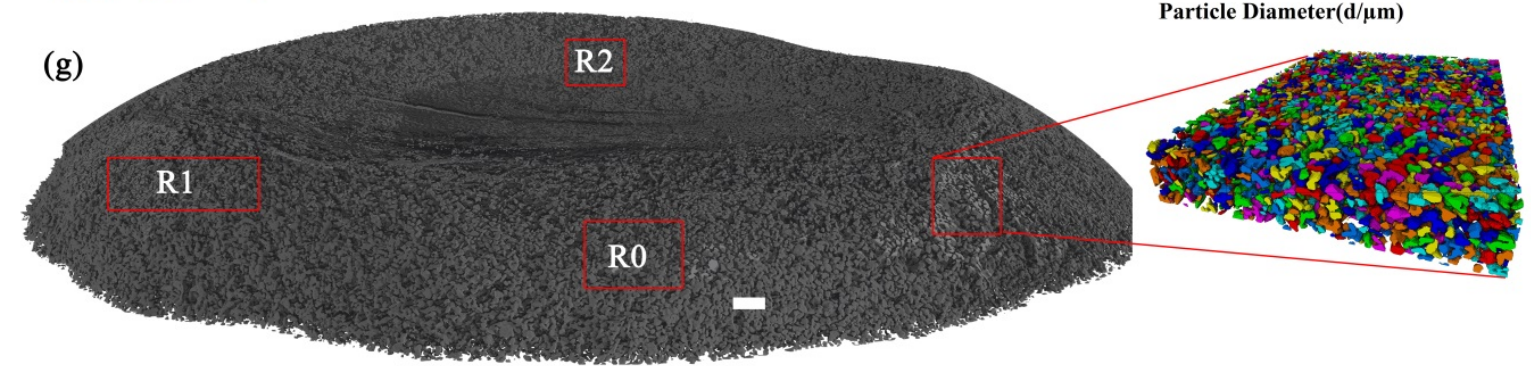

Figure 3. a) X-ray projection image of the assembled Li electrode/separator/Si electrode stack within the battery. The scale bar is $200 \mu \mathrm{m}$ long. b) Example of a reconstructed grayscale slice image. c) Same slice after binarization. d) Slice after separation and labeling. e) Scanning electron micrographs (SEM) of the mixed Si and carbon. Scale bars in b) - e) are $50 \mu \mathrm{m}$ long. f) Particle size distribution (PSD) obtained from the labeled particles (red) compared to values measured by laser diffraction (black). g) 3D visualization of all Si particles with labeled regions R0, R1 and R2. The scale bar is $100 \mu \mathrm{m}$ long.

Gas evolution is directly observed in 3D after each discharge or charge step and the reconstructed gas evolution is straightforwardly correlated to the electrochemical process. During data processing, we can easily separate the gas evolved of the channel from the solid materials due to the near zero absorption of gas. After the first discharge process, we captured the internal morphological change by tomographic imaging. In Figure S2, different 3D views of the composite of Si electrode and gas or channel are displayed. Figure 4a shows a cropped region after the first discharge, labeled as R0 in Figure 3g, in which Si particles are shown in gray and gas or channel in green. We then charge the battery and obtain the first charged state tomogram. The corresponding 3D renderings are displayed in Figure S2b and SM 2b and the corresponding cropped region is shown in Figure $4 \mathrm{~b}$. In the final step, we discharge the battery again and acquire the second discharged state tomogram shown in Figure S2c, SM 2c and Figure 4c. In addition, during the discharge and charge process, we simultaneously capture the gas movement inside the battery in 2D by in situ X-ray radiography. The results are shown in Figure 5 and SM 3. 


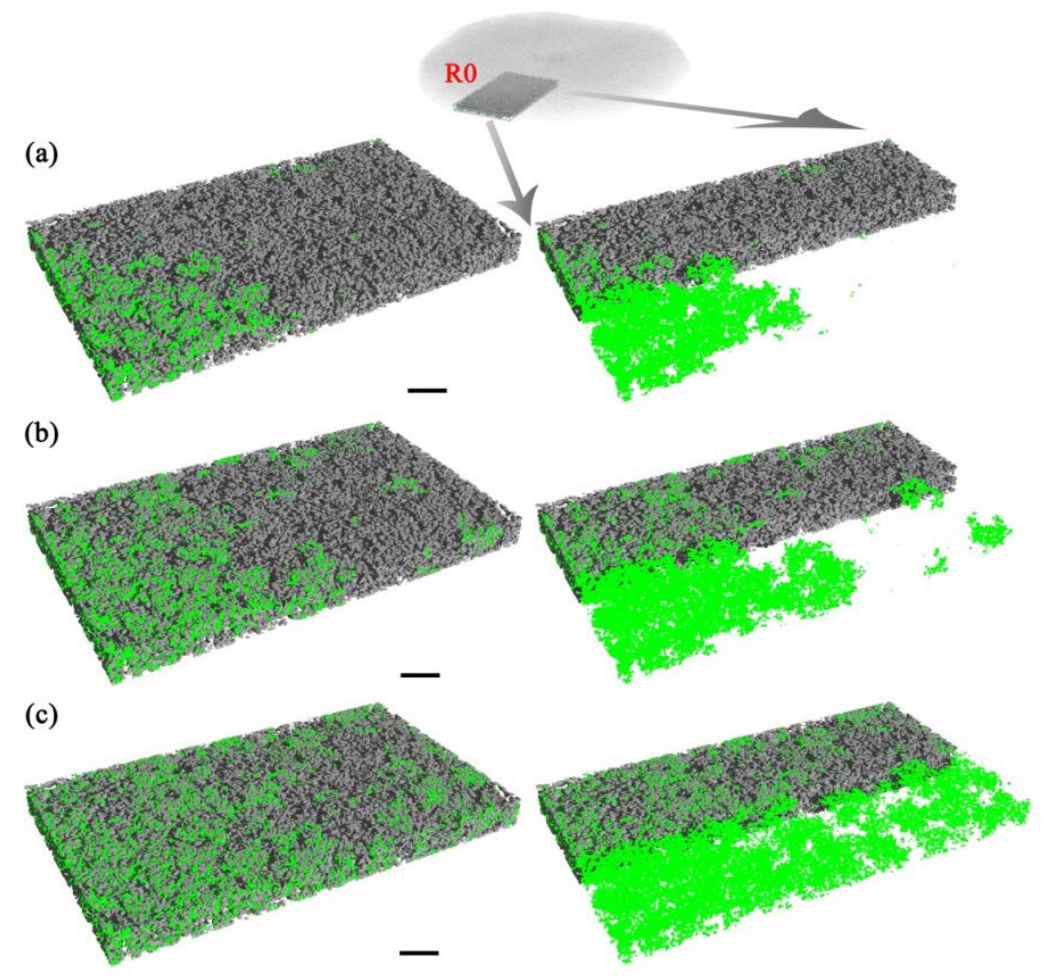

Figure 4. 3D visualization of gas evolution in a cutout at position R0 in the battery after each discharge or charge process (see Figure 3g). a) After the first discharge. b) After the first charge. c) After the second discharge. In all pictures, from left to right is from the periphery to the central part of the electrode. The left column shows the Si particles (gray) and the gas or channel (green) in a composite image. In the right column, one half of the particles is rendered transparent to show the gas part. The scale bar is $100 \mu \mathrm{m}$ long in all the pictures. 


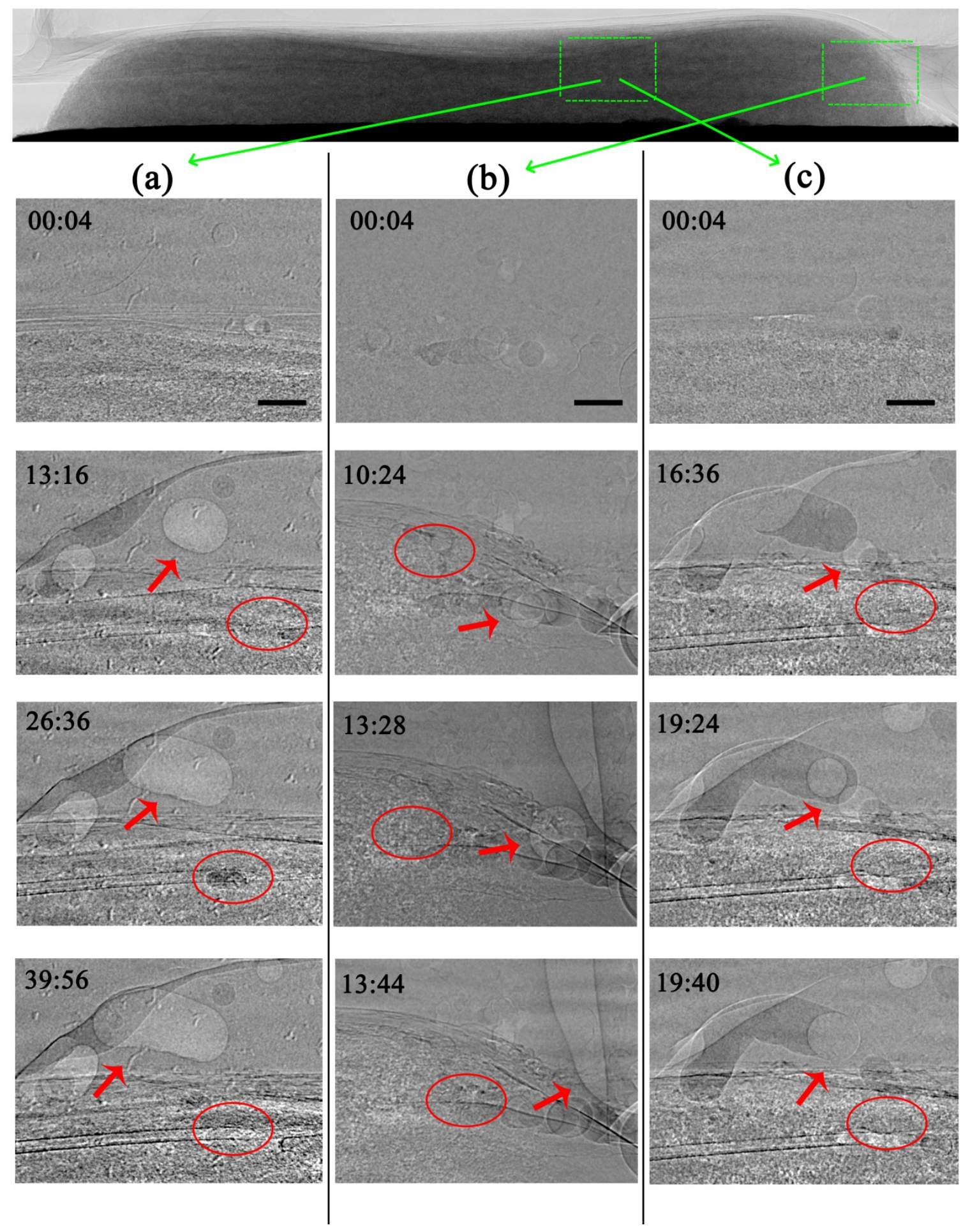

Figure 5. A series of projections showing the movement of gas as imaged by X-ray radiography. a) First discharge step. b) First charge step. c) Second discharge step. The scale bar is $100 \mu \mathrm{m}$ long in all the pictures. Ellipses mark areas of gas generation, arrows point at areas where gas bubbles have moved to. Columns a) and c) are in the middle, column b) near the edge of the electrode. See more in SI and SM 3.

During cycling, lithium ions will pass through the separator, reduce or oxidize the electrolyte on the surface of the electrode, form a solid electrolyte interface (SEI) layer containing a large amount of generated gas, and finally lithiate or delithiate the electrode ${ }^{49}$. In 
Figure S2, Figure 4, and Figure 5 as well as in the in operando 2D movie SM 3, it is clearly shown that a pronounced gas generation and accumulation takes place within the electrode during the first discharge step, which is consistent with previous experimental results. ${ }^{15,50}$ However, some phenomena were observed that are in contradiction to what had been assumed previously. One is the non-uniform distribution of gas generation sites. During electrolyte reduction/oxidization by active lithium, on the one hand, the reductive/oxidative decomposed products form the SEI layer with organic carbonate outer surface and an inner inorganic salt ${ }^{45}$ fully covering the electrode. On the other hand, notable amounts of gas are released. The polymer SEI layer remains on the surfaces of the electrodes and to some extent shapes into the wall of the channels through which the newly generated gas readily migrates out and finally merges with the accumulated gas, which eventually displaces the electrolyte and then takes the form of a skeleton structure within the electrode as clearly shown in Figure S2a and Figure 4a. Actually, because of the higher flow of electrochemically reactive Li ions it is plausible to find larger gas agglomerations close to the separator and wide channels grow into the electrode from the separator, which is consistent with the literature. ${ }^{18}$ However, there are also a large number of gas bubbles or channels that are generated in the periphery of the electrode. The reason for this may stem from the high $\mathrm{Li}$ ion concentration in the electrolyte, which surrounds the electrode. ${ }^{51}$ Another reason may stem from the Si electrode morphology that can modify the distribution of the electric field in the electrode. As shown in Figure 3a and g, the surface of the Si electrode is not flat, with the circumferential edge slightly higher than the center. Actually, it has been simulated that the electric filed has its highest value in the electrolyte at the tip of lithium dendrites. ${ }^{52}$ In our case, the non-flat Si electrode morphology may result in an inhomogeneous electric field, with higher intensity in the circumferential edge than the center area. As a result, the lithium ions, driven by the different electric field, will preferentially react with the electrode materials in the periphery region. In the region near the current collector, the development of gas bubbles or channels is also observed, most likely because the Li ions are transported via the contacted particles ${ }^{53}$ or by interstitials or vacancies in the SEI. ${ }^{54}$ In a series of radiographic images during the first discharge process, see Figure $5 \mathrm{a}$ and SM 3a, we can clearly observe the gas movement. Figure $5 b$ and SM $3 b$ along with Figure 5c and SM 3c show the first charge and the second discharge process, respectively. They also clearly reveal unexpected dynamics of gas evolution and channel development during the electrochemical process. Note that radiographic images might give misleading information on the direction of gas movement because of the ignored third dimension. In contrast, the full view of gas evolution in 3D provided by tomography allows us access the three dimensional channel structures.

Another unexpected phenomenon is the continuous production of gas in successive cycles. Contrary to the widespread belief that lithium ions diffuse uniformly from the separator to the current collector and form a lithiation front extending through the entire electrode, ${ }^{55}$ the bubbles evolve successively from the circumferential surface into the bulk of the inner electrode and form a branched system of gas bubbles and channels. It follows from the observed gas evolution after delithiation or lithiation - as clearly shown in Figure S2b-c and Figure 4b-c -that lithium ions preferentially enter the electrode in an inwardly radial passage. The direct full-view visualization of the three-dimensional gas evolution presented here suggests diffusion pathways of lithium ions inside the anode material that are non-uniformly distributed.

In fact, recently more and more attentions are being devoted to the investigation of the electrochemically active particles in relation to the overall electrode current and/or the degree of current homogeneity. ${ }^{24-25,} 56$ For example, a discrepancy is observed between electrochemical measurements that represent the overall state of the cell and spectroscopic 
data that represent a local state. ${ }^{57-58}$ A recent experiment by Delmas et al. showed the coexistence of fully lithiated and fully delithiated individual $\mathrm{LiFePO}_{4}$ cathode particles by Xray diffraction after full lithiation. ${ }^{59}$ Sugar et al. and Brunetti et al. further concluded that at any given time during cycling, only a small fraction of the total ensemble of particles is actively charged or discharged. ${ }^{57,60} \mathrm{Li}$ et al. conducted an in-depth characterization and observed that only $5 \%$ to $8 \%$ of particles are actively intercalating during lithiation, while during delithiation the active population ranges from 8 to $32 \% .{ }^{24}$ They further confirm that the current is heterogeneously distributed in the electrode, that is to say, only a small number of active particles carries most of the current regardless of the total electrode current. Taking into account that lithiation of Si will not occur until the native silicon oxide is at least partially reduced by $\mathrm{Li}$ ions ${ }^{49}$ and the gas evolution behavior presented here, we assume that diffusion of Li ions takes place as follows: during lithiation, Li ions that pass though the separator and/or the native $\mathrm{Li}$ ions in the native lithium salt electrolyte will first diffuse to the potentially active electrode particles, reduce the electrolyte and/or lithium salts with the obtain of electrons from the electrode and successively reduce the native silicon oxide, thus forming the SEI layer with inorganic inner species and organics outer species, releasing gas, and finally lithiate the active particles of the electrode, leaving non-active local regions intact. ${ }^{10}$ Vice versa during delithiation. Using this picture, we can convincingly correlate gas evolution and channel formation with the active or potentially active particles during electrochemical delithiation and lithiation. More specifically, where gas is generated, where the active particles are, where the electrochemical current is concentrated. This finding is fundamental. For the first time, the spatially distributed electrochemically active particles in an operated LIB are directly visualized via the gas evolution.

For a more convenient visualization of the distributed population of active particles and/or potentially active particles, we separated them from the remaining particles. The results are shown in Figure $6 \mathrm{~b}$ and Table 1 . After each delithiation and lithiation step, the active particle population increases concurrently with the electrochemical process. From above analysis, we interpret delithiation and lithiation as a process of progressively activating potentially active particles.

Table 1. Relative gas or channel volume fraction and number of active particles (both in \%) as a function of the cycle state in region 1 and region 2 (see Figure 3g).

\begin{tabular}{|l|c|c|c|}
\hline $\begin{array}{l}\text { Fraction State } \\
\text { Name First discharge }\end{array}$ & First charge & Second discharge \\
\hline $\begin{array}{l}\text { Region } \\
\text { Gas/Channel }\end{array}$ & 20 & 31 & 39 \\
\hline $\begin{array}{l}\text { Region } \\
\text { Gas/Channel }\end{array}$ & 14 & 20 & 39 \\
\hline $\begin{array}{l}\text { Region 1 Active } \\
\text { Particles }\end{array}$ & 29 & 46 & 64 \\
\hline $\begin{array}{l}\text { Region 2 Active } \\
\text { Particles }\end{array}$ & 18 & 29 & 57 \\
\hline
\end{tabular}




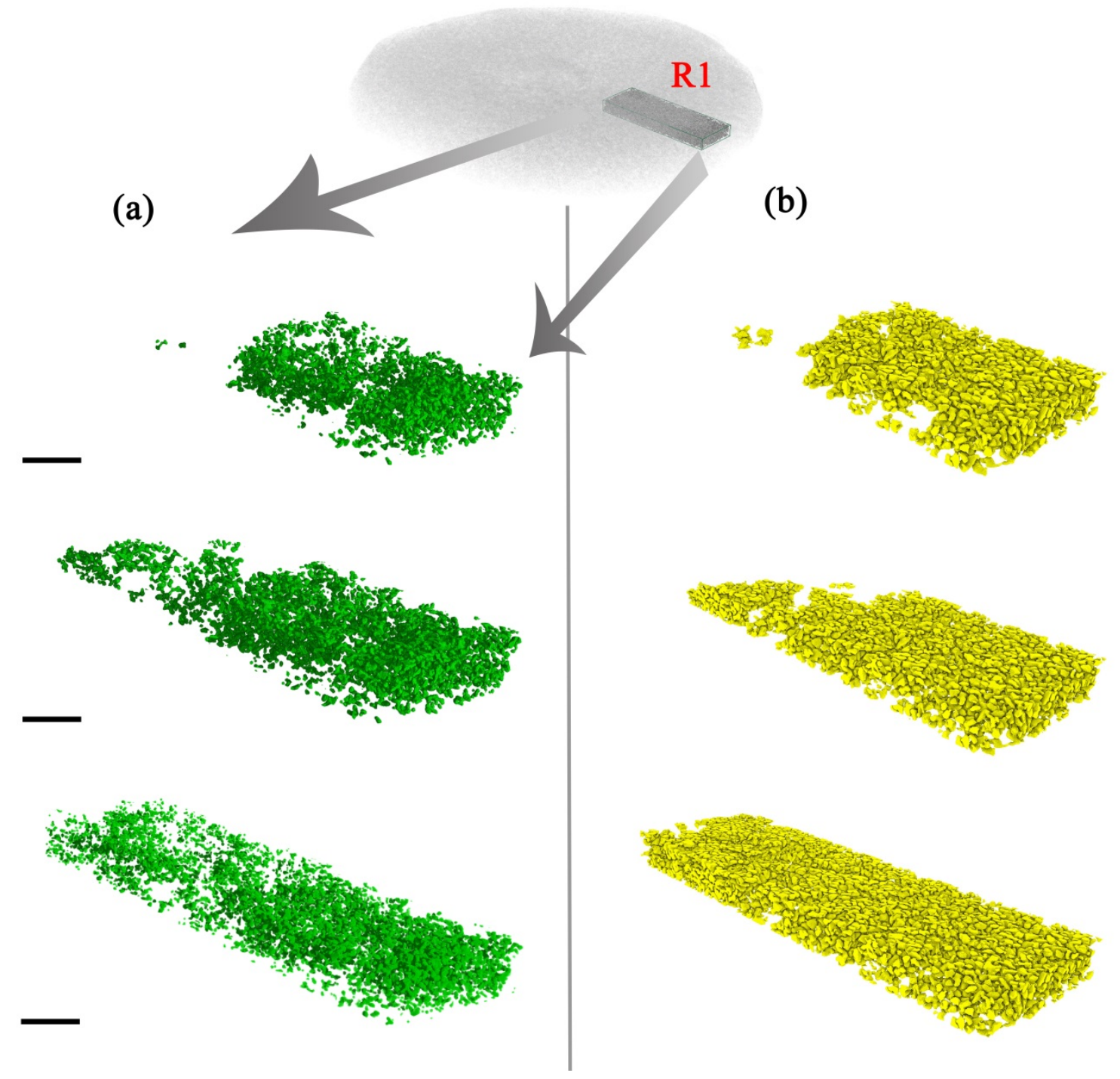

Figure 6. 3D visualization of region1as defined in Fig. 3g. a) gas or channel. b) active particles. From top to bottom: after first discharge, after first charge, after second discharge. The scale bar is $100 \mu \mathrm{m}$ long in all the pictures.

To further quantify the relationship between both gas or channel volume changes and the population of active particles with electrochemical reactions, we calculated the volume fraction of gas or channel volume and the fraction of active particles after each charge and discharge process. Two independent regions - labeled R1 and R2 in Figur 3g - are extracted for quantitative analysis. The evolution of the gas or channel volume in region $\mathrm{R} 1$ is presented in Table 1 and in Figure 6a. After the first discharge step, the fraction of gas or channel volume is only $20 \%$ compared to $31 \%$ after the first charge step. After the second discharge step, the gas or channel volume fraction is $\sim 39 \%$. Along with these changes of gas or channel volume fraction, the increase of the fraction of active particles is evident in Figure $6 \mathrm{~b}$ and Table 1, from $29 \%$ in the first discharged state to $46 \%$ in the first charged state and $64 \%$ in the second discharged state. Both trends imply the aforementioned delithiation/lithiation activation mechanism that progresses in each cycle. The analysis of R2 yields a similar result, increasing confidence in the applicability of the mechanism to the entire electrode. 
The experiment conducted here connects the gas bubble or channel evolution with the evolution of effectively or potentially active particles in a functional LIB. This opens new perspectives in optimizing the overall performance of a battery. From the viewpoint of electrode engineering, it is important to find optimal battery operating conditions or to develop new materials with reduced gas formation. There are already some reports of adding electrolyte additives ${ }^{8,10}$ and using new electrolytes ${ }^{13}$ to control gas generation and increase Li ion transport rates to enhance the LIBs's reversible performance. It is also crucial to optimize the electrode architecture to significantly increase the population of active particles to sustain the overall galvanostatic current and at the same time to improve the homogeneity of the distribution of active particles to prevent the extent of shocks and fractures induced by high local currents. ${ }^{24}$ From the perspective of numerical simulation, it is important to identify the impact of SEI formation with the associated gas release within the electrode on the transport properties of $\mathrm{Li}$ ions and cycling performance of LIBs during discharge and charge ${ }^{12}$ to further speed up the optimization of electrode structures. Altogether, the three dimensional microscale investigations of gas in the present study contribute to the understanding of the complex discharge and charge processes.

\section{CONCLUSIONS}

Novel insights into the spatial distribution and kinetics of gas evolution are unraveled by 3D synchrotron X-ray imaging for the first time. The knowledge obtained here enriches our understanding of the electrochemical activity in a real functional LIB. The gas evolution and channel formation occurring in a functional LIB have been directly visualized in a full-3D view after discharge and charge. Gas channels successively evolve from the surface of the anode material into the bulk of the electrode and form a branched system of gas bubbles and channels. The observed gas evolution points at an activation of individual particles progressing from cycle to cycle. These novel findings presented here also highlight the unique synchrotron X-ray characterization tool for revealing underlying mechanisms of LIBs and shed light on a whole range of processes that could not previously be characterized in 3D. Indeed, our results and the value of information we obtained should speed up the optimization of the architecture and/or material of the current LIBs to design more stable electrodes to further enhance their performance for next-generation demand.

\section{ACKNOWLEDGEMENTS}

The assistance of the beamline scientist of the BAMline, Dr. Heinrich Riesemeier, is gratefully acknowledged. We thank Norbert Beck for fabricating the beamline battery and Elkem AS for providing us with Si particles. This work is sponsored by the HelmholtzZentrum Berlin and China Scholarship Council.

\section{ASSOCIATED CONTENT}

Supporting Information Available: including Methods, Data Processing, Figure S1-S3 and captions for the Supporting Movies. This material is available free of charge via the Internet at http://pubs.acs.org. 


\section{REFERENCES}

(1) Yuk, J. M.; Seo, H. K.; Choi, J. W.; Lee, J. Y. Anisotropic Lithiation Onset in Silicon Nanoparticle Anode Revealed by in Situ Graphene Liquid Cell Electron Microscopy. ACS Nano 2014, 8, 7478-7485.

(2) Lin, F.; Markus, I. M.; Nordlund, D.; Weng, T.-C.; Asta, M. D.; Xin, H. L.; Doeff, M. M. Surface Reconstruction and Chemical Evolution of Stoichiometric Layered Cathode Materials for Lithium-Ion Batteries. Nat Commun 2014, 5, 3529-3538.

(3) Sun, F.; Huang, K.; Liu, Y.; Gao, T.; Han, Y.; Zhong, J. Hierarchical Structure of Co3o4 Nanoparticles on Si Nanowires Array Films for Lithium-Ion Battery Applications. Appl. Surf. Sci. 2013, 266, 300-305.

(4) Lv, D.; Shao, Y.; Lozano, T.; Bennett, W. D.; Graff, G. L.; Polzin, B.; Zhang, J.; Engelhard, M. H.; Saenz, N. T.; Henderson, W. A.; Bhattacharya, P.; Liu, J.; Xiao, J. Failure Mechanism for Fast-Charged Lithium Metal Batteries with Liquid Electrolytes. Adv. Energy Mater. 2014, 5, 1400993-1401000.

(5) Sun, F.; Huang, K.; Qi, X.; Gao, T.; Liu, Y.; Zou, X.; Zhong, J. Enhanced 3d Hierarchical Double Porous Co3o4/Graphene Architecture for Superior Rechargeable Lithium Ion Battery. Ceram. Int. 2014, 40, 2523-2528.

(6) Browning, K. L.; Baggetto, L.; Unocic, R. R.; Dudney, N. J.; Veith, G. M. Gas Evolution from Cathode Materials: A Pathway to Solvent Decomposition Concomitant to Sei Formation. J. Power Sources 2013, 239, 341-346.

(7) Vetter, J.; Holzapfel, M.; Wuersig, A.; Scheifele, W.; Ufheil, J.; Novák, P. In Situ Study on Co2 Evolution at Lithium-Ion Battery Cathodes. J. Power Sources 2006, 159, 277-281.

(8) Wuersig, A.; Scheifele, W.; Novák, P. Co2 Gas Evolution on Cathode Materials for Lithium-Ion Batteries. J. Electrochem. Soc. 2007, 154, A449-A454.

(9) Lanz, M.; Lehmann, E.; Imhof, R.; Exnar, I.; Novák, P. In Situ Neutron Radiography of Lithium-Ion Batteries During Charge/Discharge Cycling. J. Power Sources 2001, 101, 177-181.

(10) Seo, J. H.; Park, J.; Plett, G.; Sastry, A. M. Gas-Evolution Induced Volume Fraction Changes and Their Effect on the Performance Degradation of Li-Ion Batteries. Electrochem. Solid-State Lett. 2010, 13, A135-A137.

(11) Goers, D.; Holzapfel, M.; Scheifele, W.; Lehmann, E.; Vontobel, P.; Novák, P. In Situ Neutron Radiography of Lithium-Ion Batteries: The Gas Evolution on Graphite Electrodes During the Charging. J. Power Sources 2004, 130, 221-226.

(12) Rashid, M.; Gupta, A. Mathematical Model for Combined Effect of Sei Formation and Gas Evolution in Li-Ion Batteries. ECS Electrochem. Lett. 2014, 3, A95-A98.

(13) Etacheri, V.; Haik, O.; Goffer, Y.; Roberts, G. A.; Stefan, I. C.; Fasching, R.; Aurbach, D. Effect of Fluoroethylene Carbonate (Fec) on the Performance and Surface Chemistry of Si-Nanowire Li-Ion Battery Anodes. Langmuir 2011, 28, 965-976.

(14) Aurbach, D.; Markovsky, B.; Shechter, A.; Ein - Eli, Y.; Cohen, H. A Comparative Study of Synthetic Graphite and Li Electrodes in Electrolyte Solutions Based on Ethylene Carbonate - Dimethyl Carbonate Mixtures. J. Electrochem. Soc. 1996, 143, 3809-3820.

(15) Kumai, K.; Miyashiro, H.; Kobayashi, Y.; Takei, K.; Ishikawa, R. Gas Generation Mechanism Due to Electrolyte Decomposition in Commercial Lithium-Ion Cell. $J$. Power Sources 1999, 81-82, 715-719.

(16) Lee, K. H.; Song, E. H.; Lee, J. Y.; Jung, B. H.; Lim, H. S. Mechanism of Gas Build-up in a Li-Ion Cell at Elevated Temperature. J. Power Sources 2004, 132, 201-205. 
(17) Agubra, V. A.; Fergus, J. W. The Formation and Stability of the Solid Electrolyte Interface on the Graphite Anode. J. Power Sources 2014, 268, 153-162.

(18) Shin, J.-S.; Han, C.-H.; Jung, U.-H.; Lee, S.-I.; Kim, H.-J.; Kim, K. Effect of Li2co3 Additive on Gas Generation in Lithium-Ion Batteries. J. Power Sources 2002, 109, 4752.

(19) Kong, W.; Li, H.; Huang, X.; Chen, L. Gas Evolution Behaviors for Several Cathode Materials in Lithium-Ion Batteries. J. Power Sources 2005, 142, 285-291.

(20) Imhof, R.; Novák, P. In Situ Investigation of the Electrochemical Reduction of Carbonate Electrolyte Solutions at Graphite Electrodes. J. Electrochem. Soc. 1998, 145, 1081-1087.

(21) Gireaud, L.; Grugeon, S.; Laruelle, S.; Pilard, S.; Tarascon, J.-M. Identification of Li Battery Electrolyte Degradation Products through Direct Synthesis and Characterization of Alkyl Carbonate Salts. J. Electrochem. Soc. 2005, 152, A850-A857.

(22) Onuki, M.; Sakata, Y.; Yanagidate, M.; Otake, Y.; Kinoshita, S.; Ue, M.; Deguchi, M. Identification of the Source of Evolved Gas in Li-Ion Batteries by Using 13c-Labeled Solvents. ECS Trans. 2008, 11, 43-47.

(23) Wang, C.-M.; Li, X.; Wang, Z.; Xu, W.; Liu, J.; Gao, F.; Kovarik, L.; Zhang, J.-G.; Howe, J.; Burton, D. J.; Liu, Z.; Xiao, X.; Thevuthasan, S.; Baer, D. R. In Situ Tem Investigation of Congruent Phase Transition and Structural Evolution of Nanostructured Silicon/Carbon Anode for Lithium Ion Batteries. Nano Lett. 2012, 12, 1624-1632.

(24) Li, Y.; El Gabaly, F.; Ferguson, T. R.; Smith, R. B.; Bartelt, N. C.; Sugar, J. D.; Fenton, K. R.; Cogswell, D. A.; Kilcoyne, A. L. D.; Tyliszczak, T.; Bazant, M. Z.; Chueh, W. C. Current-Induced Transition from Particle-by-Particle to Concurrent Intercalation in Phase-Separating Battery Electrodes. Nat Mater 2014, 13, 1149-1156.

(25) Dreyer, W.; Jamnik, J.; Guhlke, C.; Huth, R.; Moskon, J.; Gaberscek, M. The Thermodynamic Origin of Hysteresis in Insertion Batteries. Nat Mater 2010, 9, 448-453.

(26) Bale, H. A.; Haboub, A.; MacDowell, A. A.; Nasiatka, J. R.; Parkinson, D. Y.; Cox, B. N.; Marshall, D. B.; Ritchie, R. O. Real-Time Quantitative Imaging of Failure Events in Materials under Load at Temperatures above $1,600^{\circ} \mathrm{C}$. Nat Mater 2013, 12, 40-46.

(27) Moosmann, J.; Ershov, A.; Weinhardt, V.; Baumbach, T.; Prasad, M. S.; LaBonne, C.; Xiao, X.; Kashef, J.; Hofmann, R. Time-Lapse X-Ray Phase-Contrast Microtomography for in Vivo Imaging and Analysis of Morphogenesis. Nat. Protocols 2014, 9, 294-304.

(28) dos Santos Rolo, T.; Ershov, A.; van de Kamp, T.; Baumbach, T. In Vivo X-Ray CineTomography for Tracking Morphological Dynamics. Proc. Natl. Acad. Sci. U. S. A. 2014, 111, 3921-3926.

(29) Möbus, G.; Inkson, B. J. Nanoscale Tomography in Materials Science. Mater. Today 2007, 10, 18-25.

(30) Manke, I.; Banhart, J.; Haibel, A.; Rack, A.; Zabler, S.; Kardjilov, N.; Hilger, A.; Melzer, A.; Riesemeier, H. In Situ Investigation of the Discharge of Alkaline Zn-Mno2 Batteries with Synchrotron X-Ray and Neutron Tomographies. Appl. Phys. Lett. 2007, 90, 214102-214105.

(31) Haibel, A.; Manke, I.; Melzer, A.; Banhart, J. In Situ Microtomographic Monitoring of Discharging Processes in Alkaline Cells. J. Electrochem. Soc. 2010, 157, A387-A391.

(32) Shearing, P. R.; Howard, L. E.; Jørgensen, P. S.; Brandon, N. P.; Harris, S. J. Characterization of the 3-Dimensional Microstructure of a Graphite Negative Electrode from a Li-Ion Battery. Electrochem. Commun. 2010, 12, 374-377.

(33) Zielke, L.; Hutzenlaub, T.; Wheeler, D. R.; Chao, C.-W.; Manke, I.; Hilger, A.; Paust, N.; Zengerle, R.; Thiele, S. Three-Phase Multiscale Modeling of a Licoo2 Cathode: 
Combining the Advantages of Fib-Sem Imaging and X-Ray Tomography. Adv. Energy Mater. 2015, 5, 1401612-1401620.

(34) Zielke, L.; Barchasz, C.; Waluś, S.; Alloin, F.; Leprêtre, J. C.; Spettl, A.; Schmidt, V.; Hilger, A.; Manke, I.; Banhart, J.; Zengerle, R.; Thiele, S. Degradation of Li/S Battery Electrodes on 3d Current Collectors Studied Using X-Ray Phase Contrast Tomography. Sci. Rep. 2015, 5, 10921-10933.

(35) Zielke, L.; Hutzenlaub, T.; Wheeler, D. R.; Manke, I.; Arlt, T.; Paust, N.; Zengerle, R.; Thiele, S. A Combination of X-Ray Tomography and Carbon Binder Modeling: Reconstructing the Three Phases of Licoo2 Li-Ion Battery Cathodes. Adv. Energy Mater. 2014, 4, 1301617-1301623.

(36) Ebner, M.; Geldmacher, F.; Marone, F.; Stampanoni, M.; Wood, V. X-Ray Tomography of Porous, Transition Metal Oxide Based Lithium Ion Battery Electrodes. Adv. Energy Mater. 2013, 3, 845-850.

(37) Shearing, P. R.; Brandon, N. P.; Gelb, J.; Bradley, R.; Withers, P. J.; Marquis, A. J.; Cooper, S.; Harris, S. J. Multi Length Scale Microstructural Investigations of a Commercially Available Li-Ion Battery Electrode. J. Electrochem. Soc. 2012, 159, A1023-A1027.

(38) Wang, J.; Chen-Wiegart, Y.-c. K.; Wang, J. In Situ Three-Dimensional Synchrotron XRay Nanotomography of the (De)Lithiation Processes in Tin Anodes. Angew. Chem. Int. Ed. 2014, 53, 4460-4464.

(39) Chen-Wiegart, Y.-c. K.; DeMike, R.; Erdonmez, C.; Thornton, K.; Barnett, S. A.; Wang, J. Tortuosity Characterization of 3d Microstructure at Nano-Scale for Energy Storage and Conversion Materials. J. Power Sources 2014, 249, 349-356.

(40) Wang, J. Watching Microstructures in Action in Lithium-Ion Batteries. ChemElectroChem 2014, 1, 329-331.

(41) Ebner, M.; Marone, F.; Stampanoni, M.; Wood, V. Visualization and Quantification of Electrochemical and Mechanical Degradation in Li Ion Batteries. Science 2013, 342, 716-720.

(42) Liu, X. H.; Zhong, L.; Huang, S.; Mao, S. X.; Zhu, T.; Huang, J. Y. Size-Dependent Fracture of Silicon Nanoparticles During Lithiation. ACS Nano 2012, 6, 1522-1531.

(43) Sun, F.; Huang, K.; Qi, X.; Gao, T.; Liu, Y.; Zou, X.; Wei, X.; Zhong, J. A Rationally Designed Composite of Alternating Strata of Si Nanoparticles and Graphene: A HighPerformance Lithium-Ion Battery Anode. Nanoscale 2013, 5, 8586-8592.

(44) Görner, W.; Hentschel, M. P.; Müller, B. R.; Riesemeier, H.; Krumrey, M.; Ulm, G.; Diete, W.; Klein, U.; Frahm, R. Bamline: The First Hard X-Ray Beamline at Bessy Ii. Nuclear Instruments and Methods in Physics Research Section A: Accelerators, Spectrometers, Detectors and Associated Equipment 2001, 467-468, 703-706.

(45) Pereira-Nabais, C.; Światowska, J.; Chagnes, A.; Ozanam, F.; Gohier, A.; Tran-Van, P.; Cojocaru, C.-S.; Cassir, M.; Marcus, P. Interphase Chemistry of Si Electrodes Used as Anodes in Li-Ion Batteries. Appl. Surf. Sci. 2013, 266, 5-16.

(46) Nie, M.; Abraham, D. P.; Seo, D. M.; Chen, Y.; Bose, A.; Lucht, B. L. Role of Solution Structure in Solid Electrolyte Interphase Formation on Graphite with Lipf6 in Propylene Carbonate. J. Phys. Chem. C 2013, 117, $25381-25389$.

(47) Zhou, X.; Yin, Y.-X.; Wan, L.-J.; Guo, Y.-G. Self-Assembled Nanocomposite of Silicon Nanoparticles Encapsulated in Graphene through Electrostatic Attraction for Lithium-Ion Batteries. Adv. Energy Mater. 2012, 2, 1086-1090.

(48) Arlt, T.; Maier, W.; Tötzke, C.; Wannek, C.; Markötter, H.; Wieder, F.; Banhart, J.; Lehnert, W.; Manke, I. Synchrotron X-Ray Radioscopic in Situ Study of High- 
Temperature Polymer Electrolyte Fuel Cells - Effect of Operation Conditions on Structure of Membrane. J. Power Sources 2014, 246, 290-298.

(49) Schroder, K. W.; Celio, H.; Webb, L. J.; Stevenson, K. J. Examining Solid Electrolyte Interphase Formation on Crystalline Silicon Electrodes: Influence of Electrochemical Preparation and Ambient Exposure Conditions. J. Phys. Chem. C 2012, 116, 1973719747.

(50) Armstrong, A. R.; Holzapfel, M.; Novák, P.; Johnson, C. S.; Kang, S.-H.; Thackeray, M. M.; Bruce, P. G. Demonstrating Oxygen Loss and Associated Structural

Reorganization in the Lithium Battery Cathode Li[Ni0.2li0.2mn0.6]O2. J. Am. Chem. Soc. 2006, 128, 8694-8698.

(51) Nadimpalli, S. P. V.; Sethuraman, V. A.; Dalavi, S.; Lucht, B.; Chon, M. J.; Shenoy, V. B.; Guduru, P. R. Quantifying Capacity Loss Due to Solid-Electrolyte-Interphase Layer Formation on Silicon Negative Electrodes in Lithium-Ion Batteries. J. Power Sources 2012, 215, 145-151.

(52) Jana, A.; Ely, D. R.; García, R. E. Dendrite-Separator Interactions in Lithium-Based Batteries. J. Power Sources 2015, 275, 912-921.

(53) Malik, R.; Abdellahi, A.; Ceder, G. A Critical Review of the Li Insertion Mechanisms in Lifepo4 Electrodes. J. Electrochem. Soc. 2013, 160, A3179-A3197.

(54) Lu, P.; Harris, S. J. Lithium Transport within the Solid Electrolyte Interphase. Electrochem. Commun. 2011, 13, 1035-1037.

(55) Ferguson, T. R.; Bazant, M. Z. Nonequilibrium Thermodynamics of Porous Electrodes. J. Electrochem. Soc. 2012, 159, A1967-A1985.

(56) Lin, F.; Nordlund, D.; Weng, T.-C.; Zhu, Y.; Ban, C.; Richards, R. M.; Xin, H. L. Phase Evolution for Conversion Reaction Electrodes in Lithium-Ion Batteries. Nat Commun 2014, 5, 3358-3367.

(57) Brunetti, G.; Robert, D.; Bayle-Guillemaud, P.; Rouvière, J. L.; Rauch, E. F.; Martin, J. F.; Colin, J. F.; Bertin, F.; Cayron, C. Confirmation of the Domino-Cascade Model by Lifepo4/Fepo4 Precession Electron Diffraction. Chem. Mater. 2011, 23, 4515-4524.

(58) Shin, H. C.; Chung, K. Y.; Min, W. S.; Byun, D. J.; Jang, H.; Cho, B. W. Asymmetry between Charge and Discharge During High Rate Cycling in Lifepo4 - in Situ X-Ray Diffraction Study. Electrochem. Commun. 2008, 10, 536-540.

(59) Delmas, C.; Maccario, M.; Croguennec, L.; Le Cras, F.; Weill, F. Lithium Deintercalation in Lifepo4 Nanoparticles Via a Domino-Cascade Model. Nat Mater 2008, 7, 665-671.

(60) Sugar, J. D.; El Gabaly, F.; Chueh, W. C.; Fenton, K. R.; Tyliszczak, T.; Kotula, P. G.; Bartelt, N. C. High-Resolution Chemical Analysis on Cycled Lifepo4 Battery Electrodes Using Energy-Filtered Transmission Electron Microscopy. J. Power Sources 2014, 246, 512-521. 